\section{Technikfolgenabschätzung in den neuen Bundesländern}

\section{Zusammenfassende Darstellung der Ergebnisse der 97er TA-Datenbank- Erhebung für den Bereich neue Bun- desländer}

\author{
von Renate Patz, Fachhochschule Merse- \\ burg
}

Mit der TA-Datenbank des Instituts für Technikfolgenabschätzung und Systemanalyse verbinden sich eine Reihe von Zielen und Ansprüchen. Zu den aus Sicht eines TA-Akteurs hervorzuhebenden zählen der Referenzcharakter für das Gebiet der TA, tragendes und zugleich förderndes Element in der TA-Landschaft zu sein, das Vernetzen bestehender wie auch potentieller TA-Einrichtungen, die Zusammenarbeit unterschiedlicher Fachrichtungen zu unterstützen, Impulse für neue Vorhaben zu geben, Informationen zu den Auswirkungen technologischer Entwicklungen für Entscheidungsträger, Anwender wie auch "Betroffene" zur Verfügung zu stellen. Zudem kann in einem Gebiet, in dem TA bislang nicht präsent war wie in der vormaligen DDR, eine solche Dokumentation zugleich als Spiegelbild einer Entwicklung von Anbeginn an angesehen werden. Daß die Entwicklung der Technikfolgenabschätzung in den neuen Bundesländern durch zum Teil heftige Schwankungen und Wechsel gezeichnet ist, verwundert kaum angesichts grundsätzlicher, nahezu alle Bereiche und Strukturen treffende Veränderungen, noch nicht abgeschlossener Such- und Profilierungsprozesse, angesichts des Druckes ökonomischer und sozialer Probleme, aus denen wiederum resultiert, daß anderen Themen und Problemen als "ausgerechnet TA" ein höheres Gewicht beigemessen wird. Zu berücksich- tigen ist ferner, daß TA ebenso wie ganzheitliches Denken und Agieren zwar eine gewisse Akzeptanz finden, auch als notwendig angesehen werden, wenn es jedoch darum geht, diese konkret zu betreiben und umzusetzen, dann werden häufig genug Barrieren und Hemmnisse spürbar. Prof. Huisinga, als Lehrbeauftragter der Otto-vonGuericke-Universität Magdeburg beteiligte er sich an den aktuellen TA-Erhebungen, kommentierte die gegenwärtige Situation in der Weise, daß er die Feststellung von M. Dierkes, Wissenschaftszentrum Berlin für Sozialforschung, aus dessen jüngstem Buch zitiert, TA habe derzeit keine Konjunktur.

An dieser Stelle seien nun die vorliegenden Ergebnisse der Ende '97 / Anfang '98 durchgeführten TA-Datenbank-Erhebung für den Bereich der neuen Bundesländer kurz zusammengefaßt. Seit 1993 werden die Erhebungen für die TA-Datenbank in den neuen Bundesländern - und zukünftig auch für die Länder Osteuropas - im Auftrag des ITAS von der Fachhochschule Merseburg durchgeführt und ausgewertet. Eine ausführliche Darstellung und tiefergehende Bewertung der Ergebnisse der Erhebung 1997 für Gesamtdeutschland und das europäische Ausland ist für die nächste Ausgabe der TADatenbank-Nachrichten vorgesehen.

Hinsichtlich der Erhebungsmethodik gab es im Bereich der neuen Bundesländer eine Modifizierung: Wie gewohnt wurden alle in der Datenbank als noch aktive geführten Institutionen - insgesamt waren es 44 Institutionen - um eine Aktualisierung der Informationen zur Einrichtung, zu Ihren Projekten und Veröffentlichungen gebeten. Darüber hinaus erfolgte in Zusammenarbeit mit Dr. Schiffer von der Forschungsstelle Technikfolgenabschätzung der SAW Sächsische Akademie der Wissenschaften zu Leipzig eine Kopplung mit der Erhebung für eine Dokumentation zur Technikfolgenforschung in den Bundesländern Sachsen, Sachsen-Anhalt und Thüringen. Diese lehnt sich an die von der Akademie für Technikfolgenabschätzung in Baden-Württemberg erstellten Dokumentationen zur Technikfolgenforschung in Baden-Württemberg an. Zum einen waren die Informationen der 1995 erstellten Dokumentation zu aktualisieren, sie bezog sich allerdings nur auf den Freistaat Sachsen, zum anderen sollten nun auch die Bundesländer Sachsen- 
Anhalt und Thüringen in die Erhebung einbezogen werden. Sowohl die Fakultäten, Fachbereiche und Institute der Hochschuleinrichtungen der drei Bundesländer (insgesamt waren dies ca. 400 Bereiche) als auch 90 außeruniversitäre Forschungseinrichtungen erhielten im Dezember 1997 die ITAS-Erhebungsbögen zur Neuaufnahme von Institutionen, Projekten und Literatur sowie zusätzlich ein Blatt mit der Bitte um a) Hinweise auf weitere TA-relevante Einrichtungen, b) eine kurze Einschätzung, bei welchen Themenfeldern bzw. Problemen ein vorrangiger Untersuchungs- und Handlungsbedarf besteht und c) eine kurze Beurteilung, worin die größten Hemmnisse bei der Beantragung und Bearbeitung von TA-Projekten gesehen werden.

\section{Zu den Ergebnissen}

Der Rücklauf insgesamt ist als eher zögerlich einzustufen. Quantitativ läßt sich die in der Tabelle aufgeführte Bilanz ziehen, differenziert ist nach Bundesländern sowie nach Hochschuleinrichtungen und außeruniversitären Forschungseinrichtungen. Bei den Hochschuleinrichtungen haben sich TA-Aktivitäten an universitären Bereichen deutlich stärker profiliert als an Fachhochschulbereichen. Von den 44 in der TA-Datenbank geführten Einrichtungen für die neuen Bundesländer haben sich 28 an der 97er Erhebung beteiligt. Mecklenburg-Vorpommern ist in der Bilanz nicht zu finden, die Anfang der 90er Jahre ohnehin nur wenigen TAInstitutionen gibt es inzwischen nicht mehr.

Tab.: Bilanz der 97er Erhebung

\begin{tabular}{|c|c|c|c|c|c|c|c|}
\hline & \multirow{2}{*}{$\begin{array}{l}\text { Art der Ein- } \\
\quad \text { richtung }\end{array}$} & \multicolumn{4}{|c|}{ "alte" TA-Institutionen } & \multicolumn{2}{|c|}{$\begin{array}{c}\underline{\text { neu }} \text { aufgenommene } \\
\text { Institutionen }\end{array}$} \\
\hline & & Anzahl & Rücklauf & Projekte & $\begin{array}{c}\text { neue } \\
\text { Projekte }\end{array}$ & Anzahl & Projekte \\
\hline \multirow[t]{2}{*}{ Berlin (Ost) } & Hochschulen & 1 & 0 & 0 & 0 & 0 & 0 \\
\hline & außerunivers. & 9 & 4 & 6 & 0 & 0 & 0 \\
\hline \multirow[t]{2}{*}{ Brandenburg } & Hochschulen & 3 & 1 & 1 & 2 & 2 & 2 \\
\hline & außerunivers. & 5 & 5 & 15 & 9 & 2 & 1 \\
\hline \multirow{2}{*}{$\begin{array}{l}\text { Sachsen- } \\
\text { Anhalt }\end{array}$} & Hochschulen & 4 & 3 & 6 & 2 & 3 & 8 \\
\hline & außerunivers. & 2 & 2 & 5 & 3 & 0 & 0 \\
\hline \multirow[t]{2}{*}{ Sachsen } & Hochschulen & 12 & 8 & 11 & 5 & 6 & 10 \\
\hline & außerunivers. & 7 & 4 & 25 & 7 & 4 & 6 \\
\hline \multirow[t]{2}{*}{ Thüringen } & Hochschulen & 0 & 0 & 0 & 0 & 9 & 14 \\
\hline & außerunivers. & 1 & 1 & 0 & 0 & 3 & 5 \\
\hline \multirow{2}{*}{$\begin{array}{l}\text { NBL } \\
\text { zusammen }\end{array}$} & Hochschulen & 19 & 12 & 18 & 9 & 20 & 34 \\
\hline & außerunivers. & 15 & 16 & 51 & 19 & 9 & 12 \\
\hline \multicolumn{2}{|l|}{ Gesamt } & 44 & 28 & 69 & 28 & 29 & 46 \\
\hline
\end{tabular}

Zum einen ist aus dieser Bilanz eine Stagnation, wenn nicht sogar ein Rückgang der TAAktivitäten bei den "alten" TA-Institutionen (also der bereits in der Datenbank geführten Einrichtungen) ablesbar. Dem ist noch hinzuzufügen, daß von den sich zurückgemeldeten Einrichtungen die GfT Gesellschaft für Technikfolgenabschätzung (I 354*) ihre Auflösung mitgeteilt hat und der Bereich Praktische Philosophie an der Universität Leipzig (I 451) seine TA-relevanten Aktivitäten mit der Berufung von Prof. Hubig an die Universität Stuttgart auch dorthin verlagert hat.

Überwiegend aus der SAW-Erhebung resultiert wiederum, daß 17 TA-Institutionen und 34 TA-Projekte neu in die TA-Datenbank auf- 
genommen werden konnten. Bei der SAWErhebung gab es ca. 70 Rückmeldungen, weitere sind noch zu erwarten. Die Ergebnisse dieser Erhebung werden in die oben erwähnte Dokumentation zur Technikfolgenforschung in Sachsen, Sachsen-Anhalt und Thüringen aufgenommen, sie wird derzeit zusammengestellt.

Auffallend ist der Aufwärtstrend in Thüringen. Die Wirkung einer gezielten TAFörderung läßt sich wohl kaum deutlicher belegen. Das Thüringer Ministerium für Wissenschaft, Forschung und Kultur (I 508) hat über die Förderrichtlinie zur "Durchführung von Forschungsprojekten zur Untersuchung von Folgen, Verträglichkeit und Akzeptanz anwendungsnaher Forschung und technologischer Entwicklungen mit unmittelbarer regionaler Relevanz" bisher insgesamt 11 Vorhaben von 10 Einrichtungen bewilligt. Aus diesem Pool resultiert die Neuaufnahme von 8 Thüringer TA-Institutionen mit 9 TA-Projekten in die TADatenbank.

Auch wenn dies ein Lichtblick in der ostdeutschen TA-Landschaft ist, der Abstand zur westdeutschen ist insgesamt noch beträchtlich. Bemerkbar macht sich das Fehlen "starker" TAInstitutionen in den neuen Bundesländern, auch, daß sich Großforschungszentren kaum diesem Thema zuwenden. Die geringe Personalstärke der TA-Institutionen in den neuen Bundesländern (überwiegend weniger als 5 Mitarbeiter) mindert zudem die Chance auf Bewilligung von TA-Projekten. Einen Ausweg aus dieser eigentlich nicht allzu ermutigenden Situation sehen eine Reihe von Einrichtungen in einer institutionellen Vernetzung, sei es in der Kooperation mit etablierten TA- und anderen Einrichtungen nicht nur der alten Bundesländer oder über regionale Netzwerke. Als Beispiele könnten hier genannt werden die Berliner Kommission Technikgestaltung und Bewertung (I 505), in der eine Reihe von TA-Akteuren mitwirken, die enge Verbindungen zum Büro für Technikfolgen-Abschätzung beim Deutschen Bundestag (TAB) pflegt, zum anderen wirkt sich aber auch deren Anbindung an die Ingenieur- und Wirtschaftsakademie Johann Beckmann e.V. auf die Verbindung zu regionalen bis hin zu osteuropäischen Einrichtungen stabilisierend aus. Eine in gewissen Grenzen vergleichbare Situation ist bei der Kommission Technikfolgenabschätzung der Sächsischen
Akademie der Wissenschaften zu Leipzig (I 543) zu verzeichnen, wobei sich diese Kommission aus der Arbeit der Technikwissenschaftlichen Klasse der Akademie heraus entwickelt hat. In diese Kommission wurden Vertreter mit TA-Kompetenz und TA-Erfahrung aus den Hochschulen Sachsens, Sachsen-Anhalts und Thüringens, des Sächsischen Staatsministeriums für Wissenschaft und Kunst sowie der Akademie für Technikfolgenabschätzung in Baden-Württemberg berufen. Weiteres Beispiel ist das BUFZ Brandenburgisches Umweltforschungszentrum (I 546). Im BUFZ sind Einrichtungen aus Brandenburg aber auch Berlin Ost und West vernetzt, die auf umweltrelevanten und TA-relevanten Gebieten arbeiten, wobei die TA-Aktivitäten im Rahmen des Arbeitskreises Technikfolgenabschätzung des Landes Brandenburg koordiniert werden. Die technikphilosophischen Bereiche der Brandenburgischen TU Cottbus (I 538 und I 600) pflegen eine enge Zusammenarbeit mit der Europäischen Akademie zur Erforschung von Folgen wissenschaftlich-technischer Entwicklungen in Bad Neuenahr-Ahrweiler. Schließlich resultieren aus der Kooperation des Zentrums für Interdisziplinäre Technikforschung (I 443) an der TU Dresden u.a. mit der SAW und mit der Friedrich-Ebert-Stiftung nicht nur gemeinsame TA-Veranstaltungen sondern auch neue TAVorhaben.

Wirft man nun einen Blick auf die Themen- und Problemfelder der bisherigen und neuer TA-Vorhaben, so lassen sich einige Schwerpunkte feststellen:

$\mathrm{Da}$ sich mehrere Einrichtungen mit der Institutionalisierung von TA bzw. dem Aufbau von TA-Netzwerken befassen, ist angesichts der Situation der TA-Landschaft in den neuen Bundesländern eigentlich auch naheliegend. Erwähnt seien hier die schon genannten Dokumentationen zur Technikfolgenforschung 1995 in Sachsen (P 2652) und 1998 in Sachsen, Sachsen-Anhalt und Thüringen (P 2652), die Projekte P 2695 und P 2696 zum Aufbau eines TA-Netzwerkes in Brandenburg (abgeschlossen 1996 bzw. 1997) von ÖkoConsens/BUFZ. In gewisser Weise können hier auch Projekte des Institutes für Wirtschaftsforschung Halle (I 255) eingeordnet werden, wie das Vorhaben "Regionale Innovationscluster als Wachstumspotentiale? Das Beispiel der neuen Bundeslän- 
der" (P 3098). Nicht zuletzt stehen in dieser Reihe die Arbeiten in der eigenen Projektgruppe Technikfolgenabschätzung, bis 1997 an der Martin-Luther-Universität Halle-Wittenberg, nun weitergeführt an der Fachhochschule Merseburg.

In engem Zusammenhang mit Fragen der Institutionalisierung stehen solche der Weiterentwicklung von TA-Methoden oder von Bewertungsverfahren, sowohl allgemeiner Art als auch themenspezifisch. So befaßte sich das BUFZ mit der "Erarbeitung eines Entscheidungsrasters für Technikfolgenabschätzungen" (P 2699, abgeschlossen 1996). Das Institut für Agrartechnik Bornim e.V. arbeitet an Bewertungsmethoden bezogen auf die landwirtschaftliche Produktion, beispielsweise "Bewertung Informationstechnik Pflanzenbau" (P 2183), "Bewertung nachwachsender Energieträger auf Grenzstandorten landwirtschaftlicher Produktion" (P 2704), "Integrierte Bewertung von Verfahren der Nutztierhaltung" (P 3067), "Entwicklung eines Simulationsmodells zur Bewertung von Strategien der Unkrautbekämpfung im Ackerbau" (P 3069). Das Zentrum für Interdisziplinäre Technikforschung an der TU Dresden bezieht sich auf die Ableitung und Untersuchung von "Kriterien und Indikatoren für eine nachhaltige Entwicklung der Wasserversorgung und des Gewässerschutzes im Freistaat Sachsen" (P 3076) bzw. das Gebiet "Kreislaufwirtschaft und Nachhaltigkeit" (P 2648).

Im Zusammenhang mit den Erhebungen für die TA-Datenbank und die Dokumentationen zur Technikfolgenforschung, aber auch darüber hinaus lebt immer wieder die Frage auf, wie TA von anderen Methoden, Verfahren abzugrenzen sei, was das spezifische von Technikfolgenabschätzungen, Technikfolgenbewertungen u.dgl. sei. Etwas verwundert, daß die Bedeutung der Gestaltung von Diskursen oder der Moderation von Bewertungs- und Entscheidungsprozessen in Diskussionen immer wieder hervorgehoben wird, Gegenstand konkreter TAProjekte in den neuen Bundesländern sind sie aber nicht. Demgegenüber spielen (technik)ethische oder philosophische Fragen, mehrfach auch in Verbindung mit der Nachhaltigkeitsdebatte, eine schon größere Rolle. Zu verweisen wäre hier auf die Projekte "Technikethik / angewandte Ethik" (P 2182) am Lehrstuhl für
Praktische Philosophie der Universität Leipzig, nun in der Abteilung Wissenschaftstheorie und Technikphilosophie an der Universität Stuttgart. "Technikfolgenbeurteilung und Wissenschaftsethik in Ländern Mittel- und Osteuropas" (P 3242) ist ein Vorhaben, das vom Arbeitsbereich Allgemeine Technikwissenschaft an der Brandenburgischen TU Cottbus in Kooperation mit der Europäischen Akademie Bad Neuenahr bearbeitet wird.

Breiten Raum nehmen begleitende TAUntersuchungen zu aktuellen technischen bzw. technologischen und gesellschaftsrelevanten Entwicklungen aus den Bereichen Verkehr, Bio- und Gentechnologie oder Forst- und Landwirtschaft ein, wobei diese häufig einzelne Auswirkungsbereiche tiefer ausloten und weitere relevante nur anreißen. Als ein Schwerpunktthema kristallisieren sich insbesondere die IuKTechnologien heraus. Es kann hier nur auf einige Projekte verwiesen werden, wie "Virtuelles Klassenzimmer. Möglichkeiten und Gefahren" (P 2655) vom Bereich Künstliche Intelligenz an der TU Chemnitz, "Telelearning zwischen elektronisch vernetzten Schulen" (P 3079) an der Pädagogischen Hochschule Erfurt, "Analyseund Gestaltungshilfen zur humanorientierten Anwendung moderner IuK-Technologien" (P 3220) am Fachgebiet Arbeitswissenschaften der TU Ilmenau oder "Sicherheit in der Kommunikationstechnik" (P 3240) am Institut für Theoretische Informatik der TU Dresden.

Betrachtet man die Entwicklungen in den neuen Bundesländern im Kontext zum eingangs erwähnten gesellschaftlichen Wandel, so wäre zu erwarten, daß sich TA-Vorhaben auch auf die Vorbereitung und Begleitung beispielsweise von Großvorhaben beziehen, die so nur mit der Wende möglich waren, wie die großen Verkehrsprojekte, die Entwicklungen im suburbanen Raum mit den ausgedehnten, oft genug überdimensionierten Handels-, Gewerbe- und Dienstleistungszentren auf der nun nicht mehr grünen Wiese. Derartige Entwicklungen als Gegenstand von TA-Projekten finden sich kaum und wenn, dann geht es um ausgewählte Teilaspekte, beispielsweise im Zusammenhang mit der Sanierung, Rekultivierung und Gestaltung von Bergbaufolgelandschaften wie sie am Institut für Bergbaufolgelandschaften Finsterwalde (I 506) untersucht werden. 
Es gibt immer wieder auch Fragestellungen, die an sich nicht neu sind, in der Verbindung mit TA jedoch die Überlegungen in Richtungen lenken, die so noch nicht verfolgt wurden, die weitere Impulse für die Technikfolgenforschung erwarten lassen. $\mathrm{Zu}$ dieser Kategorie kann das Projekt "Zukunft des Wissens" bzw. Wissen für die Zukunft (P 2702) am Lehrstuhl für Technikphilosophie der Brandenburgischen TU gerechnet werden.

Die hier genannten Themen- und Problemfelder als Gegenstand von TA-Untersuchungen geben lediglich einen Ausschnitt wider. Als knappes Resümee könnte folgendes gezogen werden: Auch wenn die TA-Landschaft in den neuen Bundesländern noch vergleichsweise dünn besiedelt ist, es zeichnen sich Kristallisationskerne, Netzwerkknoten ab. TA hat keine Konjunktur, das kann im Resultat der 97er TADatenbank-Erhebung partiell auch gefolgert werden, würde aber den - trotz oder gerade wegen der gegebenen und nicht gerade einfachen Rahmenbedingungen - sichtbaren Aktivitäten den Boden und die Motivation für weiterführende entziehen. Es wäre nun eher zu fragen, welche Konsequenzen sich aus solch einer Feststellung ableiten lassen bzw. auch real abgeleitet werden?

Dieser Beitrag sei abschließend Gelegenheit, um allen zu danken, die sich an der Erhebung beteiligt haben, die uns ihre Meinung zur TA mitgeteilt haben und es seien diejenigen um Nachsicht gebeten, die wir möglicherweise bei der Erhebung übersehen haben. In diesem Falle bitten wir Sie, auch von sich aus den Kontakt zu uns zu suchen.

\section{Anmerkung}

* Bei den I- bzw. P-Nummern handelt es sich um die Zugriffsnummern, unter denen die Informationen zu den genannten Institutionen bzw. Projekten in der TA-Datenbank zu finden sind. Siehe auch die "Hinweise zur Nutzung der TADatenbank" am Ende dieses Heftes.

\section{Kontakt}

Dr. Renate Patz

Fachhochschule Merseburg, Forschung und

Technologietransfer / Technikfolgenabschätzung

Geusaer Straße, D-06217 Merseburg

Tel.: + 49 (0) 3461/462907, -2903

Fax: + 49 (0) 3461/462919, -2365

E-mail: renate.patz@ltg.fh-merseburg.de 